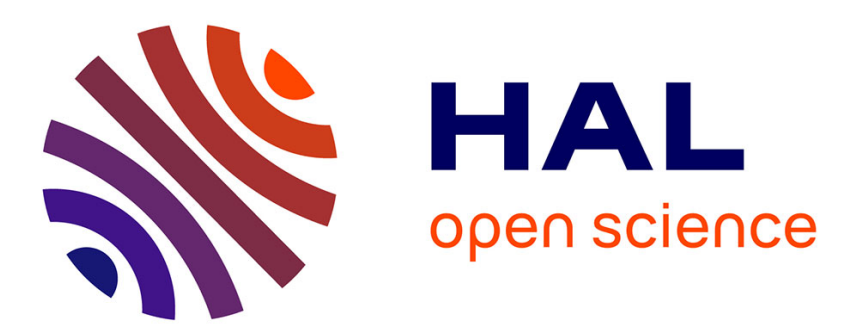

\title{
Enhancing the Field of View Limitation of Visible Light Communication-based Platoon
}

\author{
Mohammad Abualhoul, Mohamed Marouf, Oyunchimeg Shagdar, Fawzi \\ Nashashibi
}

\section{- To cite this version:}

Mohammad Abualhoul, Mohamed Marouf, Oyunchimeg Shagdar, Fawzi Nashashibi. Enhancing the Field of View Limitation of Visible Light Communication-based Platoon. 6th International Symposium on Wireless Vehicular Communications: WIVEC2014, Sep 2014, Vancouver, Canada. hal-01007692

\section{HAL Id: hal-01007692 \\ https://hal.inria.fr/hal-01007692}

Submitted on 17 Jun 2014

HAL is a multi-disciplinary open access archive for the deposit and dissemination of scientific research documents, whether they are published or not. The documents may come from teaching and research institutions in France or abroad, or from public or private research centers.
L'archive ouverte pluridisciplinaire HAL, est destinée au dépôt et à la diffusion de documents scientifiques de niveau recherche, publiés ou non, émanant des établissements d'enseignement et de recherche français ou étrangers, des laboratoires publics ou privés. 


\title{
Enhancing the Field of View Limitation of Visible Light Communication-based Platoon
}

\author{
Mohammad Y. Abualhoul, Mohamed Marouf, Oyunchimeg Shagdar and Fawzi Nashashibi \\ \{mohammad.abu_alhoul, mohamed.marouf, oyunchimeg.shagdar, fawzi.nashashibi\}@inria.fr
}

\begin{abstract}
Visible Light Communication (VLC) technology have recently been suggested as efficient supportive technology for platooning applications over short inter-vehicle distances. Though, ensuring the continuity of Line-of-Sight (LOS) of any optical-based applications is one of the most complex scenarios for an autonomous vehicle control, and still remains as an open challenge for Intelligent Transformation Systems (ITS). Exchanging information about the relative directional position of each member of the platoon, together with front and rear facing directions of each vehicle, can be very useful data for building a smooth geometrical-based compensation method, which results in ensuring that any increase in both incidence and irradiance optical angles will never exceed the Field of View (FOV) limitations and regardless of the trajectory shape. This paper propose a tracking alike compensation method of four vehicles equipped with positioning and VLC systems. We have simulated different scenarios related to different trajectories and compensation angles limits. The simulation results show that trajectories influence on the optical incidence and irradiance angles can be compensated efficiently and without deploying any tracking method.
\end{abstract}

\section{INTRODUCTION}

$\mathbf{N}$ OWADAYS deployment of wireless technologies for Intelligent Transformation Systems (ITS), have triggered a tremendous wireless spectrum demand. As the standardized Radio Frequency (RF) spectrum for vehicular applications is getting tight and expensive, the push toward more wireless bandwidth is driving wireless technologies into alternative and supportive spectrum bands. In the mean time, Light Emitting Diode (LED) has become very common in automotive lighting due to its long service life, high resistance to vibration, and better safety performance due to its short rise time. Therefore, it is possible to use these LED lightning infrastructures as communications equipments and construct VLC system with remarkably low installation costs.

Besides the many advantages of using VLC for ITS applications, this technology can be provided without considering regulations, interference, and the most favorable advantage of VLC-based applications for ITS is to perform communication functionality while maintaining the main function of the LED as illumination source [1].

A number of existing studies employ LEDs that already exist in vehicles, such as brake lights, turn signals and headlamps, to carry out Vehicle-to-Vehicle (V2V) and Infrastructure-Vehicle (I2V) communications [2] [3]. Among the many possible VLC-based applications for ITS, platooning support is one of attractive applications due to the relatively short communication link requirements and the type of the exchanged data [4].

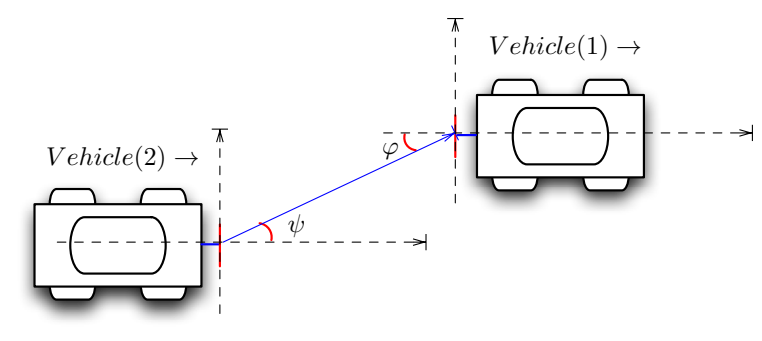

Fig. 1. Optical communication-based platooning system for V2V using rare light model, where $\psi$ and $\varphi$ represents the both incidence and irradiance angles.

The remainder of the paper is organized as follows. After brief description of the problem in Section II, a review of our previous work together with related work is presented in Section III. The study of VLC limitation and the compensation method is discussed in Section IV. Section VI is dedicated to the performance evaluation and analytical simulation results of the VLC-based platoon performance. A comparison of the result obtained from a SIMULINK enhanced model and the results in [4] will be also presented. Finally, we conclude the paper in Section VII.

\section{Problem Description}

In our previous study [4], we investigated analytical model suggesting to employ the vehicles commercial rear lights as a reliable communication link. Ambient noise and the FOV limitations were the main two challenges may prevent using VLC for any ITS application and specifically for VLC-based platooning. Many solutions were proposed to overcome the ambient noise presence [5] for the case of platoon, where the communication link is relatively the short range inter-vehicle distance between platoon members. Even thought, FOV which is expressed as the maximum angular size of the PhotoDiode (PD) remained as the main challenge when VLC-based platoon members follow sharp trajectories. We aim to present a tracking alike method using independent optical arm controller solution in order to enhance the FOV limitation. The proposed method compensating the increase in the both incidence and irradiance angle, smoothly and in real time. This method will insure the condition of the described angles in Fig. 1 for stable VLC link as the following:

$$
0 \leqslant\left(\begin{array}{lll}
\psi & \& \& & \varphi
\end{array}\right) \leqslant \theta_{c}
$$


where $\psi$ and $\varphi$ represent the incidence and irradiance angular variation, and $\theta_{c}$ is the maximum critical FOV angle.

\section{RELATED WORK}

Over the last years, a remarkable number of projects such as PATH in California [6], PRAX-ITELE [7] and SARTRE [8] in Europe have dealt with the platoon concept as an approach to increase the traffic safety and the road throughput on urban area and highways. In most of the platoon projects, RF-based technology was always the communication solution to exchange information between platoon members. Although, most of the RF-based communication technologies which share the same spectrum are considered to facilitate relatively long range and high data rate communication for vehicular applications, these technologies still suffer from RF channel congestion in highly dense vehicular traffic scenarios [9],

New methods involving optical communication have been lately considered for relatively short range applications in ITS. As a previous contribution, we studied the analytical modeling of VLC for platoon application, where we built full analytical model in order to clarify and define the main parameters that have significant impact on the outdoor VLC system performance [4]. We also developed a longitudinal and lateral platooning controllers based on V2V VLC communication. The longitudinal controller aims to maintain a constant intervehicle distance while the lateral controller aims to follow the trajectory of the head vehicle.

D.-R. Kim et al. [10] tested an outdoor VLC system based on Controller Area Network (CAN) for ITS, where the total achieved communication distance was 20 meter in the daytime outdoor condition. A VLC system suitable for outdoor applications has been developed and tested in [11], where a full performance evaluation of a new modulation scheme for VLC was introduced. Also, a remarkable effort to implement one of the first PHY and MAC layers of the VLC Standard (IEEE802.11.7) was demonstrated and tested in [12], where a half-duplex VLC prototype for ITS applications has been realized.

The authors of [13] have developed optical transmitter tracking algorithm for I2V application. The performed algorithm used wide-angle and narrow-angle cameras, combined with real time image processing in order to comeover optical receiver FOV limitations. To our best knowledge, there are no proposed solutions to overcome the FOV physical limitation for V2V optical link in ITS.

\section{VLC-Based Platoon Limitations}

The analytical study detailed in [4] shows that optical channel DC gain $H(0)$ for ITS platoon application can be modeled as LED Lambertian emission, considering the both incidence $(\psi)$ and irradiance $(\varphi)$ angles geometry as in Fig. 1.

$$
H(0)=\left\{\begin{array}{cc}
\frac{(m+1) A_{p h}}{2 \pi d^{2}} \cos ^{m}(\varphi) T_{s} g(\psi) \cos (\psi), & 0 \leqslant(\psi, \varphi) \leqslant \theta_{c} \\
0 & \text { elsewhere }
\end{array},\right.
$$

where $d$ is inter vehicle distance and $\theta_{c}$ is the Photo-Diode (PD) field of view, which indicate the maximum allowed incidence angle. $A_{p h}$ is the physical area of the PD at the receiver side, and $T_{s}$ is the filter transmission coefficient. For an ideal optical filter, such as the case of our previous study assumption, $T_{s}=1.0 . m$ represents the lampertian emission order as shown in (3). The chosen half-power angle $\hat{\phi}$ has a remarkable influence on the coverage range and pattern shape of the lambertian light source. By choosing narrower $\hat{\phi}$, we can directly increase the service range [14]. And finally, $g(\psi)$ is the optical concentrator gain, (see TABLE 1 in [4] for full simulation parameters details).

$$
\mathbf{m}=-\frac{\ln 2}{\ln (\cos \hat{\phi})} .
$$

By adapting the fixed inter-vehicle distance platooning model, we can see from (2) that $\theta_{c}$ is the key parameter specifying the physical limitation of the VLC-based platoon. The most crucial drawback addressed in [4] was concerning FOV limitation, where the scenario of sharp curvature (Trajectory 2) as depicted in Fig. 2 resulted in non-line of sight mode which is totally signal loss mode in VLC-based systems. This signal loss mode of VLC-based platoon is expressed by safety stop of the second member of the platoon which cause full stop for all the platoon member.

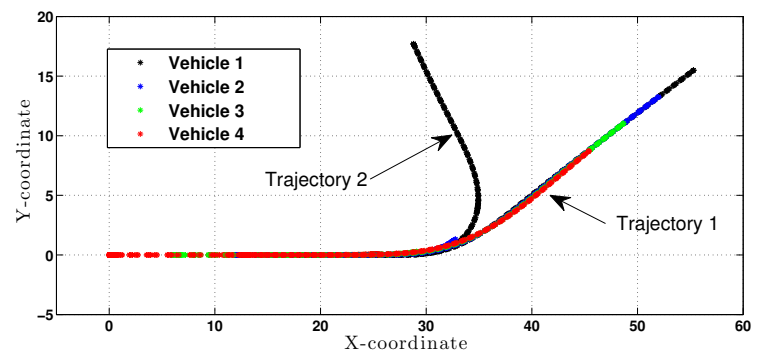

Fig. 2. Four vehicles platoon model in SIMULINK for two trajectories Trajectory 1 illustrate the success scenario, when the queued vehicles fall in the FOV of each other. Trajectory 2 illustrate the failure scenario when the trajectory curvature will cause out of FOV (Extracted results from [4]).

\section{Proposed Solution to Enhance FOV Limitations}

Exchanging information about the relative directional position of each member of the platoon, together with front and rear facing directions of each vehicle, can be very useful data to build a smooth geometrical-based compensation method. This exchanged information can be processed in each vehicle controller, and generate a real time compensation, ensuring that the increase in both incidence and irradiance angles will never exceed the FOV limitation for any trajectory.

Figure 3 shows a simple platoon formation consist of two vehicles, where $R$ represents the mounted optical receiver/transmitter arm length on each vehicle, $l$ and $d$ are the vehicle length and the inter-vehicle distance between the both vehicles respectively. The angles between vehicles axis and 


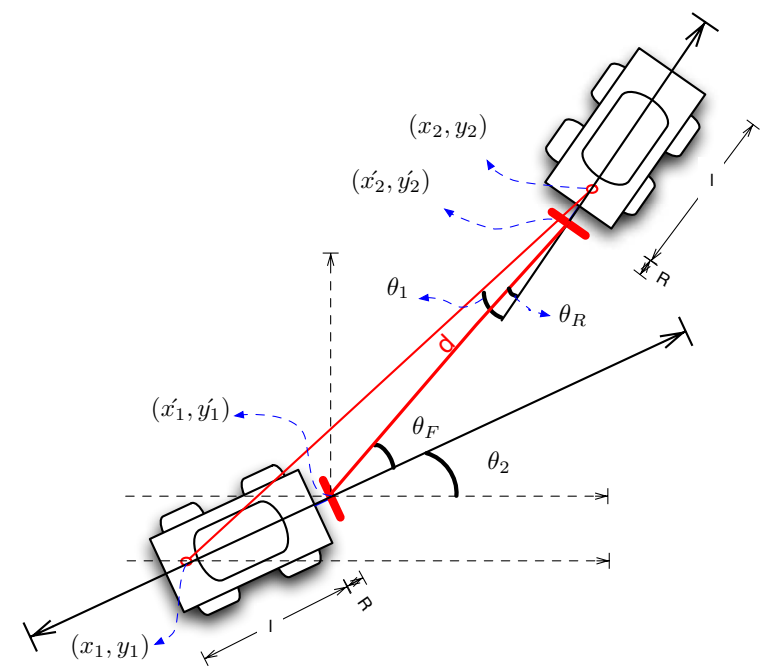

Fig. 3. Two vehicles VLC-based platoon ray tracing model.

the reference $\mathrm{x}$-axis for the addressed vehicles are $\theta_{2}$ and $\theta_{1}$, where $\theta_{F}$ and $\theta_{R}$ represents the both front and rear angles.

As in any communication-based platoon, we assume that each vehicle is equipped with a positioning system located in the positions $\left(x_{2}, y_{2}\right)$ and $\left(x_{1}, y_{1}\right)$ as depicted in Fig 3. The direct LOS distance $d$ can be calculated by defining the relative coordinates $\left(x_{1}^{\prime}, y_{1}^{\prime}\right)$ and $\left(x_{2}^{\prime}, y_{2}^{\prime}\right)$, which represents the coordination of the mounted optical receiver and transmitter.

$$
\begin{gathered}
\left\{\begin{array}{l}
x_{1}^{\prime}=x_{1}-R * \cos \left(\theta_{1}\right) \\
y_{1}=y_{1}-R * \sin \left(\theta_{1}\right)
\end{array},\right. \\
\left\{\begin{array}{l}
x_{2}^{\prime}=x_{2}+(l+R) * \cos \left(\theta_{2}\right) \\
y_{2}^{\prime}=y_{2}+(l+R) * \sin \left(\theta_{2}\right)
\end{array} .\right.
\end{gathered}
$$

These relative coordinates can be expressed as two relative distance victors in both $\mathrm{x}$ and $\mathrm{y}$ coordinates,

$$
\left\{\begin{array}{l}
x_{r e l}=x_{1}-x_{2}^{\prime} \\
y_{r e l}=y_{1}-y_{2}^{\prime}
\end{array},\right.
$$

where the square law of those two relative vectors, is the shortest LOS distance between the mounted LED on the rear of vehicle 1 and the PD mounted on the front of vehicle 2 .

$$
d=\sqrt{\left(x_{r e l}\right)^{2}+\left(y_{r e l}\right)^{2}} .
$$

Using the result from (6), the front angle of the second vehicle can be expressed as the following:

$$
\theta_{F}=\operatorname{atan}\left(\frac{y_{r e l}}{x_{r e l}}\right),
$$

while the rear angle of the head vehicle is:

$$
\theta_{R}=\theta_{1}-\left(\theta_{F}+\theta_{2}\right)
$$

After defining the relations between the vehicles orientation and the both front and rear angles $\left(\theta_{R}, \theta_{F}\right) \rightarrow f\left(\theta_{2}, \theta_{1}\right)$, we can express the optimal compensation value for both incidence and irradiance angles as the following:

$$
\left\{\begin{array}{c}
\psi_{x}=\alpha \theta_{F} \\
\varphi_{x}=-\alpha \theta_{R}
\end{array}\right.
$$

where $\alpha$ represent the whole platoon direction by comparing between the head and the follower relative coordinates, which allows the mounted optical component arms controller to define the compensation direction if clockwise or counterclockwise.

$$
\left\{\begin{array}{ccc}
\alpha=+1, & y_{2}>y_{1} & \text { Counterclockwise } \\
\alpha=0, & y_{2}=y_{1} & \text { No-compensation } . \\
\alpha=-1, & y_{2}<y_{1} & \text { Clockwise }
\end{array}\right.
$$

As can be verified by the simulations in Section VI, a real time compensation of the both irradiance and incidence angles, at the same time inheriting the smooth orientation change of the both head and tail vehicle, will expand the FOV limitation condition for stable VLC link as the following:

$$
0 \leqslant\left(\psi_{\&} \varphi\right) \leqslant \theta_{c}+\theta_{\text {arm }}
$$

where $\theta_{\text {arm }}$ is the maximum angle which can be achieved by tuning the arms in Fig 3 with respect to the vehicles axises and depending on the arm length.

\section{Performance Evaluation}

Through this section, the limitation of the ordinary FOV, together with the proposed enhancement method simulation results are presented for different trajectories and different applied $\theta_{\text {arm }}$ to each member of the platoon. The variation of the both $\theta_{F}$ and $\theta_{R}$ were analyzed, where the influence of the compensation incidence and irradiance angels is observed and compared with the results in [4].

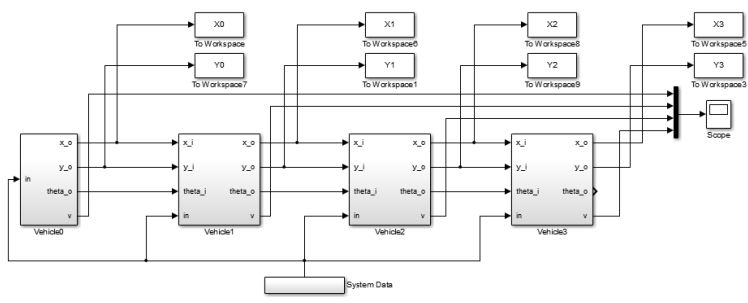

Fig. 4. VLC-based Platoon SIMULINK model for four vehicles, each vehicle consist of longitudinal controller, lateral controller, VLC model for path loss calculation and an a FOV enhancment controller.

Assuming that each vehicle knows its position, and the preceding vehicle will relay the required information to the next one in the queue using VLC link, we simulate a platoon of four vehicles have an initial inter-vehicle distance of 4 meters and a constant speed of $20 \mathrm{~m} / \mathrm{s}$, which falls in the range of the stable communication link offered by the proposed VLC model for platooning application. Fig. 4 illustrates four vehicles VLCbased platoon SIMULINK model, where each block contain a vehicle longitudinal and lateral controller, together with a VLC channel model. In addition to the SIMULINK model presented in [4], we added an independent compensation arms-controller and sharper trajectories generator. 


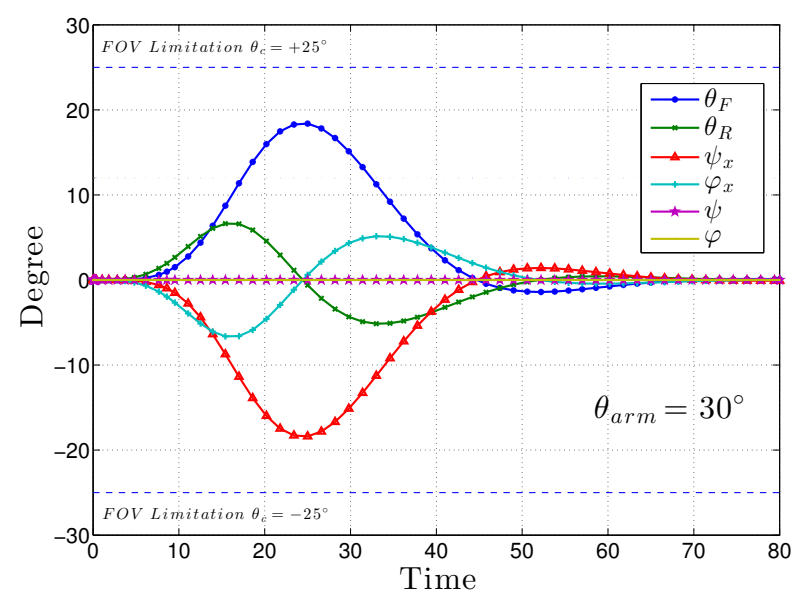

Fig. 5. The angular variation of the vehicle front $\left(\theta_{F}\right)$, vehicle rear $\left(\theta_{R}\right)$, incidence compensation angle $\left(\psi_{x}\right)$, irradiance compensation angle $\left(\varphi_{x}\right)$, incidence angle $(\psi)$ and irradiance angle $(\varphi)$ between every two members in the VLC-based platoon and following trajectory 2 in Fig 6.

As soon as the variation in both $\theta_{F}$ and $\theta_{R}$ which are expressing the trajectory sharpness dose not exceed the value of $\theta_{\text {arm }}$, the compensation method works smoothly by applying the compensation angles $\psi_{x}$ and $\varphi_{x}$ and results in zero value for both $\psi$ and $\varphi$. This result is not only extending the FOV limitation and ensure the continuity of the LOS between the platoon members, but it is also remarkably improve the optical path loss, where the two terms $\cos ^{m}(\varphi)$ and $\cos (\psi)$ in (2) will have no influence on the optical DC Gain calculation.

$$
H(0)=\left\{\begin{array}{cc}
\frac{(m+1) A_{p h}}{2 \pi d^{2}} T_{s} g(\psi) & 0 \leqslant(\psi \& \& \varphi) \leqslant \theta_{c} \\
0 & \text { elsewhere }
\end{array}\right.
$$

Figure 5 shows selected simulation results of the smooth compensation method, and based on the exchanged information of the relative orientation and positioning of each member of the platoon. The results are expressing the trajectories 2 in Fig. 8 and Fig. 7, where $\theta_{F}$ is never exceeding $\theta_{\text {arm }}$ all along the platoon path, and result in full compensation.

The scenario where $\theta_{F}$ or $\theta_{R}$ is exceeding $\theta_{\text {arm }}$ can be simulated either by reducing $\theta_{\text {arm }}$ or applying sharper curvature. Figure 6 shows the smooth build up in $\psi$ for compensation angle limit of $30^{\circ}$, while the front angle of the second vehicle is reaching up to $58^{\circ}$. The build up in $\psi$ is a result of adapting the trajectory 3 in Fig 7. Although that $\psi$ is increasing for such sharp trajectories, the limitation condition of FOV is still maintained all along the trajectory path for all the platoon member, with slight increment of the optical signal path loss.

Comparing the results illustrated in Fig. 8 and Fig. 7 is enough to understand the impact of extending the FOV limitation for VLC-based platoon. Trajectory 2 and 3 in Fig. 7 caused a total signal loss scenario for any optical communication-based platoon system, which was the defined limits of the optical receiver in [4]. Applying the proposed enhancement method to the same scenarios and dealing with the

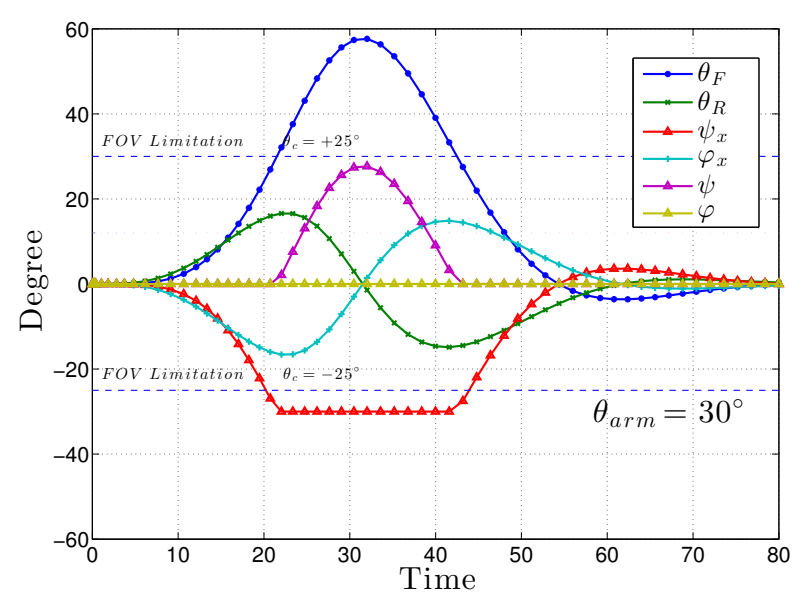

Fig. 6. The angular variation of the vehicle front $\left(\theta_{F}\right)$, vehicle rear $\left(\theta_{R}\right)$, incidence compensation angle $\left(\psi_{x}\right)$, irradiance compensation angle $\left(\varphi_{x}\right)$, incidence angle $(\psi)$ and irradiance angle $(\varphi)$ between every two members in the VLC-based platoon and following trajectory 3 in Fig 6

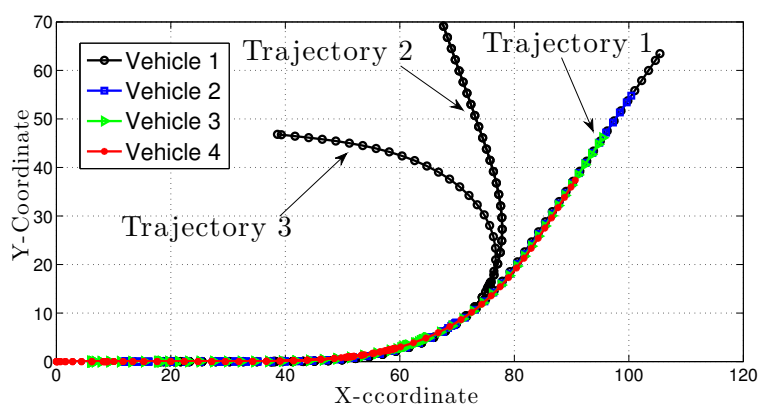

Fig. 7. Four vehicles platoon model in SIMULNK for three different trajectories. Trajectory 1 illustrate the success scenario when the queued vehicle fall in the FOV of each other. Trajectory 2 and 3 illustrate the failure scenario before applying the enhancement method and when the $\theta_{F}$ or $\theta_{R}$ are exceeding $\theta_{c}$ limits.

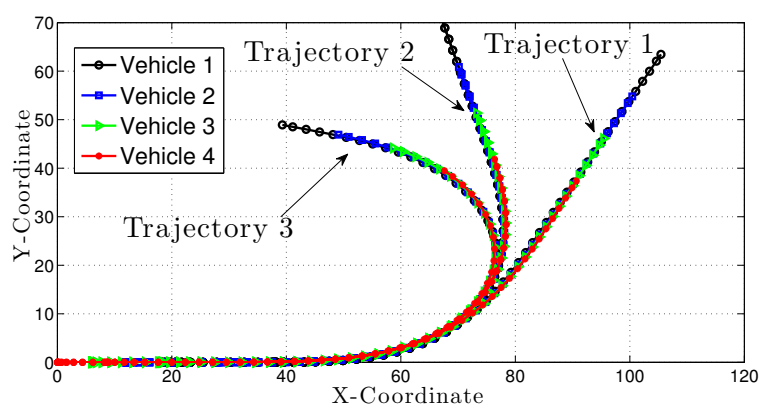

Fig. 8. Four vehicles platoon model in SIMULNK for three different trajectories. Trajectory 1,2 and 3 illustrate the success scenario when the FOV enhancement method is applied, where $\theta_{F}$ and $\theta_{R}$ never exceeding $\theta_{c}$.

same physical limitations of the parameters in the mentioned study, results in an officiant compensation of the both $\psi$ and $\varphi$.

The simulation results for many other different scenarios 
performed using our proposal, confirmed that VLC-based platoon is no longer limited to the receiver FOV, while choosing the right value for $\theta_{\text {arm }}, \theta_{c}$ will enable the VLC-based platoon members to overcome any trajectory as soon as the presence of blocking obstacle is not assumed.

\section{CONCLUSION}

This paper introduced a tracking alike solution of VLCbased platooning platform, where we tackled the problem of a VLC-based autonomous platoon going through sharp curvatures and intersection areas. The simulation results showed that our proposed method was able to compensate the trajectories influence on the optical incidence and irradiance angles efficiently and without deploying any tracking method. Smooth and real time response was observed in our simulation outputs and resulted in remarkable reduction of the total optical path loss between any two members of the platoon. For further research, we plan to study the effects of VLC-based platoon acceleration limits, mobility and vehicles vibration that expected to have influence on the both optical received signal and FOV enhancement method.

\section{REFERENCES}

[1] R. D. Dupuis and M. Krames, "History, development, and applications of high-brightness visible light-emitting diodes," Journal of Lightwave Technology, vol. 26, no. 9, pp. 1154-1171, May 2008.

[2] M. Yoshino, S. Haruyama, and M. Nakagawa, "High-accuracy positioning system using visible LED lights and image sensor," in 2008 IEEE Radio and Wireless Symposium, Jan. 2008, pp. 439-442.

[3] J.-H. Yoo, R. Lee, J.-K. Oh, H.-W. Seo, J.-Y. Kim, H.-C. Kim, and S.-Y. Jung, "Demonstration of vehicular visible light communication based on LED headlamp," in 2013 Fifth International Conference on Ubiquitous and Future Networks (ICUFN), Jul. 2013, pp. 465-467.

[4] M. Abualhoul, M. Marouf, O. Shagdar, and F. Nashashibi, "Platooning control using visible light communications: A feasibility study," in 2013 16th International IEEE Conference on Intelligent Transportation Systems - (ITSC), Oct. 2013, pp. 1535-1540.

[5] I. Lee, M. Sim, and F. Kung, "Performance enhancement of outdoor visible-light communication system using selective combining receiver," IET Optoelectronics, vol. 3, no. 1, pp. 30-39, Feb. 2009.

[6] S. Shladover, "Path at 20 - history and major milestones," in Intelligent Transportation Systems Conference, 2006. ITSC '06. IEEE, 2006, pp. 122-129.

[7] P. Daviet and M. Parent, "Longitudinal and lateral servoing of vehicles in a platoon," in Intelligent Vehicles Symposium, 1996., Proceedings of the 1996 IEEE, 1996, pp. 41-46.

[8] E. C. Tom Robinson, Eric Chan, "Operating platoons on public motorways: An introduction to the sartre platooning programme," in 17th World Congress on Intelligent Transport Systems (ITS) 2010, October 2010.

[9] S. Eichler, "Performance evaluation of the IEEE 802.11p WAVE communication standard," in Vehicular Technology Conference, 2007. VTC2007 Fall. 2007 IEEE 66th, 2007, pp. 2199-2203.

[10] D.-R. Kim, S.-H. Yang, H.-S. Kim, Y.-H. Son, and S.-K. Han, "Outdoor visible light communication for inter- vehicle communication using controller area network," in 2012 Fourth International Conference on Communications and Electronics (ICCE), Aug. 2012, pp. 31-34.

[11] N. Lourenco, D. Terra, N. Kumar, L. Alves, and R. L. Aguiar, "Visible light communication system for outdoor applications," in 2012 8th International Symposium on Communication Systems, Networks Digital Signal Processing (CSNDSP), Jul. 2012, pp. 1-6.

[12] A. Belle, M. Falcitelli, M. Petracca, and P. Pagano, "Development of IEEE802.15.7 based ITS services using low cost embedded systems," in 2013 13th International Conference on ITS Telecommunications (ITST), Nov. 2013, pp. 419-425.
[13] S. Okada, T. Yendo, T. Yamazato, T. Fujii, M. Tanimoto, and Y. Kimura, "On-vehicle receiver for distant visible light road-to-vehicle communication," in 2009 IEEE Intelligent Vehicles Symposium, Jun. 2009, pp. $1033-1038$

[14] J. Kahn and J. Barry, "Wireless infrared communications," vol. 85, no. 2, pp. $265-298$, Feb. 1997. 